\title{
NUEVA YORK: ¿CIUDAD COMPACTA Y SUSTENTABLE?
}

\author{
Tom AngoTti \\ Profesor de Urban Affairs and Planning, City University of New York \\ ASTRAGALO, 20 (2015) \\ Attribution-NonCommercial-ShareAlike - CC BY-NC-SA \\ Artículo, ISSN 2469-0503 \\ https://dx.doi.org/10.12795/astragalo.2015.i20.05
}

\section{PLANYC2030 Y EL MITO DE LA CIUDAD SUSTENTABLE}

Mi objetivo principal aquí es desmitificar la noción, ampliamente difundida por los medios internacionales, que la ciudad de Nueva York representa un modelo de la ciudad sustentable, en gran parte debido a su alta densidad. El crecimiento urbano en los EE.UU. durante el siglo pasado ha sido ampliamente criticado por ser de muy baja densidad y los urbanistas consideran la ciudad compacta de Europa como una alternativa, pero típicamente Nueva York es reconocida como la excepción a la regla, tal vez la máxima ciudad compacta en USA. El mito dice que por su alta densidad Nueva York es un modelo y un gran desafío al sprawl -el desarrollo metropolitano disperso y horizontal. La crítica del sprawl ha sido particularmente aguda entre urbanistas y ambientalistas ${ }^{1}$.
El mito de Nueva York como excepción fue afianzado recientemente a partir del anuncio del PLANYC2030 en 2007 bajo el mandato del entonces alcalde Michael Bloomberg, (City of New York, 2007) ${ }^{2}$.El plan, bajo el lema A Greener, Greater New York establece muchos objetivos cuantificables para mejorar el ambiente, pero la razón de ser principal del plan es el objetivo de crear viviendas para una nueva población de más de un millón de personas, principalmente en nuevos centros de desarrollo de alta densidad. El plan y los sucesivos cambios en la reglamentación urbana consideran que la presencia en Nueva York del sistema de transporte público más grande de USA ofrece muchas oportunidades de promover un desarrollo urbano más eficiente y sostenible, en particular alrededor de las estaciones de tránsito donde hay potencial para aumentar la densidad bajo la estrategia llamada transit-oriented development. 
Un informe de la Regional Plan Association (2012) recomienda la densificación, promoviendo proyectos de gran escala. Dos libros recientes ${ }^{3}$ adoptan este razonamiento y avanzan la hipótesis que la ciudad de Nueva York es un modelo para USA y el mundo y a su vez Gallagher ${ }^{4}$ (2014) propone que las periferias urbanas están en crisis, aumentando la importancia de los centros.

Otro mito avanzado ampliamente por Bloomberg fue que la densificación, usando el modelo Manhattan, es la solución a la eventual alza en los niveles del mar debido al cambio climático. En la última década Nueva York sufrió dos tempestades fuertes que causaron daños debidos a inundaciones y Bloomberg inició estudios sobre la protección de las costas, reformas de los códigos urbanos y una propuesta de construir un gran centro inmobiliario nuevo, elevado sobre el futuro nivel del mar, en el sur de Manhattan, llamado Seaport City ${ }^{5}$.

Los medios fueron casi unánimes en situar a Bloomberg y Nueva York en la vanguardia de políticas públicas con respecto al cambio climático pero hay dudas sobre la factibilidad de tales propuestas y muchas críticas porque el proyecto vuelve la espalda al resto de la ciudad que estaría más expuesta a las posibles inundaciones ${ }^{6}$.

Al contrario del mito de Nueva York como ciudad densa y sustentable quiero demostrar que el crecimiento del Manhattan vertical en el último siglo sigue siendo insustentable, ineficiente y además promueve una ciudad desigual en un área metropolitana compleja. Ese mito oculta la realidad de un área metropolitana dispersa y desigual. En el futuro, con la prevista elevación del nivel del mar debido a los cambios climáticos la distancia entre el mito y la realidad será aún más grande y la política de densificar creará una metrópolis con el centro fortificado y protegido del mar creciente y la periferia más vulnerable a los trastornos del clima.

Aunque el PlaNYC2030 es un plan para el municipio de Nueva York --que incluye los condados de Manhattan, Brooklyn, Bronx, Queens y Staten Island- el imaginario creado en el plan es que la parte de la ciudad más densa -la isla de Manhattan- dada su relevancia en relación a la sustentabilidad frente a la elevación de la cota marina debe ser el modelo para el desarrollo futuro de la ciudad entera, el área metropolitana y hasta el resto del mundo. Este razonamiento se usa para justificar una nueva fase de desarrollo de alta densidad no sólo en Manhattan -donde el suelo disponible está ahora muy limitado- sino especialmente fuera de Manhattan, en las áreas cercanas de Brooklyn, Queens y Bronx.

Es verdad que utilizando como medida la huella de carbono, Nueva York produce menos impacto que otras ciudades de USA.: 7.1 toneladas per cápita frente 24.5 para la USA urbana $^{7}$. La cifra para Manhattan debe ser aún más baja porque la gran mayoría del dióxido de carbono introducido al ambiente se debe a la operación ineficiente en los edificios de menos unidades, y en el transporte, aspectos que son más eficientes en Manhattan por su alta densidad. La mayoría de los residentes de Manhattan no tiene automóvil y el sistema de transporte público está centrado en la isla de Manhattan.

Pero hay que reconocer ciertos problemas con el modelo que ha seguido Manhattan: El plan reticular no incluye espacios verdes, con la gran excepción de Central Park; muchas calles carecen de sol y aire fresco (el objetivo del zoning era de maximizar estos), la temperatura 
media es mucho más alta; y alrededor de $25 \%$ del suelo en la isla es vulnerable al mar creciente debido a las previsiones del cambio climático.

Glaeser y Owens creen que también Manhattan debe ser el modelo para otras ciudades en USA. y en el mundo. El primer capítulo del libro de Owens se titula Más Como Manhattan aunque esta frase representa la confusión que permite la difusión del mito: Manhattan es vertical y densa, Nueva York mucho menos y el área metropolitana es de muy baja densidad, en un contraste dramático con Manhattan.

\section{MANHATTAN VERSUS ÁREA METROPOLITANA}

El primer problema con el mito es metodológico: es importante distinguir la isla de Manhattan, la ciudad de Nueva York, y el área metropolitana de Nueva York. La metrópolis funciona como una entidad económica y social más o menos integrada en un territorio donde todos los componentes del centro y la periferia están conectados uno con el otro. Manhattan representa solo el 0.1 por ciento del territorio metropolitano y el 7 por ciento de la población. La ciudad de Nueva York representa sólo el 2\% del territorio y el $40 \%$ de la población. Entonces, el desarrollo de alta densidad en Manhattan es la excepción y no la regla.

Es cierto que en las últimas décadas se han formado, según el Regional Plan Association (www.rpa.org/), 12 nuevos centros comerciales grandes en el área metropolitana de Nueva York. Algunos de éstos demuestran características de alta densidad, con sus rascacielos y negocios en el sector financiero, y todos tienen algún vínculo de transporte público con Manhattan. Más que representar el florecimiento de nuevos núcleos urbanos independientes, estos centros expresan una expansión fuera de Manhattan de algunas funciones económicas debido al costo altísimo del suelo en Manhattan comparado a las periferias más cercanas.

Es también cierto que en las últimas décadas se han formado nuevos núcleos urbanos en toda la periferia metropolitana que rompen con la monotonía de las casas unifamiliares y la forma urbana de extrema densidad baja. Las llamadas edge cities incluyen nuevos centros de usos mixtos, y centros de investigación y producción de la alta tecnología en proximidad a residencias de tipologías variadas ${ }^{8}$.

Cuadro 1: Densidades en el Área Metropolitana de Nueva York

\begin{tabular}{|l|c|c|c|c|c|}
\hline & $\begin{array}{c}\text { Área } \\
\left(\mathrm{Km}^{2}\right)\end{array}$ & $\begin{array}{c}\text { \% Área } \\
\text { Metropolitana }\end{array}$ & $\begin{array}{c}\text { Población } \\
\mathbf{2 0 1 3}\end{array}$ & $\begin{array}{c}\text { \% Pobla- } \\
\text { ción }\end{array}$ & $\begin{array}{c}\text { Densidad } / \mathrm{km}^{2} \\
\mathbf{2 0 1 2}\end{array}$ \\
\hline $\begin{array}{l}\text { Área } \\
\text { Metropolitana }\end{array}$ & $\mathbf{3 4 4 9 0}$ & $\mathbf{1 0 0}$ & $\mathbf{2 3 5 0 8 6 0 0}$ & $\mathbf{1 0 0}$ & $\mathbf{7 2 4}$ \\
\hline Nueva York & 786 & $\mathbf{2}$ & 8405837 & 36 & 10094 \\
\hline Manhattan & $\mathbf{5 9}$ & $\mathbf{0 , 1}$ & $\mathbf{1 6 2 6 1 5 9}$ & $\mathbf{7}$ & $\mathbf{2 7 5 6 2}$ \\
\hline Brooklyn & 183 & 0,5 & 2592149 & 11 & 14164 \\
\hline
\end{tabular}




\begin{tabular}{|l|c|c|c|c|c|}
\hline & $\begin{array}{c}\text { Área } \\
\left(\mathrm{Km}^{2}\right)\end{array}$ & $\begin{array}{c}\text { \% Área } \\
\text { Metropolitana }\end{array}$ & $\begin{array}{c}\text { Población } \\
\mathbf{2 0 1 3}\end{array}$ & $\begin{array}{c}\text { \% Pobla- } \\
\text { ción }\end{array}$ & $\begin{array}{c}\text { Densidad } / \mathrm{km}^{2} \\
\mathbf{2 0 1 2}\end{array}$ \\
\hline Bronx & 109 & 0,3 & 1418733 & 6 & 13016 \\
\hline Queens & 283 & 0,8 & 2296175 & 10 & 8114 \\
\hline Staten Island & 151 & 0,4 & 472621 & 2 & 3130 \\
\hline
\end{tabular}

Fuente: US Census Bureau

La metrópolis debe ser la unidad de análisis porque representa la totalidad de la entidad urbana. La ciudad de Nueva York es una entidad política creada en 1898 cuando el territorio era principalmente rural y el centro urbanizado fue limitado a una pequeña parte de la isla de Manhattan. Ahora el área metropolitana incluye partes de tres estados y miles de entidades locales.

\section{[ver cuadro 1]}

Nueva York es una de las áreas metropolitanas más dispersas en el país. A pesar de que Manhattan tiene 27562 residentes por $\mathrm{km}^{2}$, el área metropolitana tiene una densidad de sólo 724 residentes, menos que Los Angeles y otras metrópolis grandes en el país. La mayoría de la población en el área metropolitana vive en la periferia de baja densidad. El 85\% de los desplazamientos en la periferia del área metropolitana son por automóvil; el sistema de transporte público en el área es dirigida principalmente hacia Manhattan y no tiene servicio para la mayoría de los desplazamientos dentro de la periferia, de periferia a periferia.

Además hay muchas deficiencias en el servicio de transporte público de la ciudad de Nueva York. Las primeras líneas fueron construidas a principios del siglo 20 y todas las líneas (menos una) confluyen en el centro de Manhattan. Dadas las deficiencias del sistema, y el sistema vial conectado al sistema metropolitano, 55\% de las transferencias a Manhattan se hace por auto, un fenómeno visible por la congestión de tráfico en los puentes y túneles a Manhattan y en las avenidas de toda la isla ${ }^{9}$.

Uno de los factores estructurales que habría que reconocer antes de planificar más desarrollo inmobiliario en la ciudad de Nueva York es que hay pocas posibilidades para la ampliación del sistema de transporte público sin gastos extraordinarios. El costo de construir más estaciones y líneas es altísimo y hasta ahora hay muy pocas ampliaciones previstas. La señalización en el sistema es anticuada y modernizarla requiere gastos públicos que hasta ahora no han estado disponibles. Hay una gran deficiencia en el sistema de transporte público de superficie (buses) fuera de Manhattan ${ }^{10}$. El servicio de buses en toda la ciudad es notoriamente lento debido a la congestión de tráfico y a pesar de la introducción de algunas nuevas líneas en vías preferenciales en los últimos años, no existe un sistema de BRT como, por ejemplo, en Bogotá, Curitiba, México y muchas otras ciudades menos desarrolladas.

Con estas limitaciones la mayoría de los hogares de Nueva York (excluyendo Manhattan) son dueños de uno o más automóviles y utilizan el vehículo privado para la mayoría de sus desplazamientos en la periferia. El tiempo 
promedio de la transferencia al trabajo en Nueva York es casi 45 minutos, 15 minutos más del promedio nacional. Este sistema de transporte es insustentable para mayor desarrollo urbano.

El PlaNYC responde a estas realidades con un llamado a la extensión de la red de vías ciclísticas y la ampliación de espacios públicos. Pero el PlaNYC solamente aspira a implementar el plan de vías ciclísticas aprobado en 1997 y comparado a las famosas ciudades compactas ciclísticas como Copenhagen y Amsterdam, que han estimulado fuertes reducciones en el uso de los vehículos motorizados, parece una medida simbólica. En la teoría y la práctica, las bicisendas y los espacios públicos sirven principalmente para dar más valor a las nuevas zonas de desarrollo inmobiliario, especialmente las que atraen la clase creativa.

\section{CIUDAD NUEVA Y CIUDAD EXISTENTE}

La conclusión y la base fundamental del PlaNYC2030 es que la construcción de nuevos centros de alta densidad en Nueva York es no solamente necesaria para llenar las necesidades de un millón de nuevos residentes que estarían por llegar, sino además para crear una ciudad más verde y sostenible.

No hay duda que la nueva construcción vertical será con nuevas tecnologías que conservan energía; esto es un argumento a favor de más densidad. Pero qué hacemos con la mayoría de los edificios existentes, que seguirán siendo la mayoría debido a la escasez y el alto costo del suelo urbano en Manhattan? Más de $65 \%$ de los edificios en la ciudad de Nueva York fueron construidos con sistemas de calefacción y aire-acondicionados ineficientes. Más de la mitad del dióxido de carbono que se emite en la ciudad de Nueva York es producto de estas ineficiencias. Esta situación es aún más dramática cuando consideramos el área metropolitana, donde la tipología típica es la casa unifamiliar y el medio de transporte que predomina es el automóvil, responsable por la producción de 25\% del CO2 en el ambiente.

\section{LA IMPORTANCIA DEL MERCADO INMOBILIARIO}

El dramático boom en la construcción de torres en Manhattan y sus alrededores desde inicios del siglo 21 es resultado de una nueva y voluminosa onda de acumulación de capital global en el sector financiero. Pero en el mundo de los urbanistas se justifica esta nueva escalada en la construcción monumental y vertical como si fuera una respuesta a los costos del desarrollo urbano horizontal y una estrategia para combatir la contaminación ambiental y el cambio climático, declarando que la alta densidad es más eficiente en el uso de la energía, el agua y la generación y tratamiento de residuos urbanos. Si examinamos de cerca este fenómeno hay muchas ineficiencias en estos nuevos enclaves y son núcleos urbanos altamente costosos y exclusivos. Son enclaves que dan la espalda a la gran mayoría de la metrópolis, que siguen siendo dispersas, de baja densidad, y que no disfrutan de los beneficios económicos del desarrollo vertical, beneficios que reciben la minoría de los dueños del suelo.

El desarrollo urbano de alta densidad es resultado de un exceso de capital financiero que busca lugares centrales donde el precio del suelo tiene máxima potencialidad de aumentar año tras año. En la última década, hasta el colapso 
del mercado financiero de 2007-2008, muchos de los centros urbanos recibieron masivas inversiones de capital, que corresponden al fenómeno spatial fix del capital ${ }^{11}$.

En otro trabajo ${ }^{12}$ he demostrado cómo, desde su fundación por los holandeses, Manhattan ha sido un gran imán, atrayendo capital a su destino mítico, Wall Street, capital de todo el mundo, y siempre la capital mundial inmobiliaria" En las últimas décadas del siglo pasado, con relativamente poco espacio libre para facilitar más construcción vertical, aparecieron en Manhattan nuevos mecanismos de inversión que tienen acceso a grandes superávits de capital creados en todo el mundo: real estate investment trusts, equity funds y otros instrumentos creativos que facilitaron el movimiento de capital en los grandes centros urbanos de todo el mundo. Muchos de los nuevos inversionistas formaron asociaciones y alianzas con poderosas entidades y familias locales que habían dominado el desarrollo urbano por más de un siglo.

\section{LA CIUDAD EXCLUSIVA Y DESIGUAL}

Nueva York es una de las ciudades más segregadas de USA, donde las divisiones raciales y de clase se reflejan en el marco del territorio urbano ${ }^{13}$. Durante los 20 años de las administraciones municipales de Rudolph Giuliani y Michael Bloomberg (1994-2014) reinó la política de promover al máximo el desarrollo inmobiliario. Bloomberg abiertamente promovió juntamente con el mito de la ciudad sustentable, la ciudad de lujo y la hipótesis que la atracción de más dinero ayuda a todos, incluyendo los menos a afortunados ${ }^{14}$. Pero el desarrollo nuevo causó muchos desplazamientos cuando aumentaba el precio del suelo y las rentas en las áreas centra- les que afectó en particular los residentes con ingresos limitados, los negros, los latinos y las otras minorías. Bajo el mandato de Bloomberg han habido más personas sin techo que en toda la historia de la ciudad, un promedio de más de 50000 cada noche, el 20\% de la población regularmente busca ayuda para llenar sus necesidades para comestibles y en las elecciones de 2013 Bill de Blasio ganó con un margen abrumador bajo el lema que criticaba la desigualdad: El Cuento de dos Ciudades. ${ }^{15}$

El crecimiento de la ciudad de lujo en el centro del área metropolitana, el desplazamiento de los residentes con menos recursos hacia la periferia y la expansión continua de la periferia está produciendo un posible futuro aún más desigual. Si Manhattan y el centro llegaran a ser la ciudad sustentable, eficiente y protegida de los impactos causados por los cambios climáticos, tal vez nos espera un futuro mucho más desigual y para la mayoría de la población, menos sustentable.

\section{MONUMENTOS A LA NUEVA DENSIDAD URBANA}

En lo que sigue presento cuatro casos de megaproyectos, para mostrar como en la práctica el mito de la sustentabilidad sirve más para marketing de los nuevos desarrollos en vez de base de una política general de sustentabilidad. Tales megaproyectos se caracterizan por los siguientes rasgos:

La nueva construcción privada tiende a fijar más demandas al sistema de transporte público, que tiene poca capacidad ociosa y muchas carencias en el mantenimiento, pero sin aumentar la capacidad y eficiencia del sistema. Donde se construyen nuevas líneas y estaciones 
ellas son muy costosas y benefician principalmente a los megaproyectos.

Las inundaciones después del huracán Sandy en 2012 causaron el cierre de varias líneas de transporte público y túneles a Manhattan. La restauración de los servicios costó miles de millones de dólares y todavía no está completa. La probabilidad de futuras inundaciones es alta.

La gran mayoría de las residencias y negocios en los nuevos proyectos sirven a una población con ingresos medios muy sobre la media.

Los nuevos megaproyectos son enclaves segregados del resto de la ciudad, y multiplican la fragmentación de la ciudad desigual.

Los megaproyectos propuestos en las costas bajo el nuevo régimen de control urbanístico tendrán altos costos y serán enclaves exclusivos.

Los megaproyectos contribuyen al proceso neoliberal de privatizar los espacios públicos, creando espacios públicos de propiedad privada en vez de espacios claramente públicos. Se trata de una expresión de la gentrificación urbana de muchas áreas centrales de ciudad.

Los megaproyectos desplazan áreas integradas, de usos mixtos, áreas que tienen una mezcla de industria y residencia y varias clases sociales y etnodiversidad. Se promueven los nuevos megaproyectos como si fueran una mezcla de usos, pero esto es marketing de una falsa autenticidad ${ }^{16}$.

Aunque se venden los nuevos proyectos como ejemplos de utilización de terrenos baldíos y sub-utilizados los nuevos proyectos tienen altas tasas de desocupación, debido a la especulación inmobiliaria. Además en sus alrededores, muchas viviendas permanecen abandonadas porque el precio del suelo ha subido y sus dueños están especulando en un futuro con precios aún más altos ${ }^{17}$.

\section{CUATRO CASOS: MANHATTAN Y MÁS ALLÁ}

Los cuatro casos son Hudson Yards, World Trade Center, Williamsburg y el plan Bloomberg de Densificación de Vivienda Pública. Sigue un breve análisis de cada uno con una nota sobre el espacio público en cada caso.

\section{HUDSON YARDS}

El plan incluye 60 manzanas urbanas, 200000 residencias, $190000 \mathrm{~m}^{2}$ de hoteles, $93000 \mathrm{~m}^{2}$ de comercio, una escuela pública y 8 hectáreas de espacio público. Se construirá en gran parte sobre una nueva plataforma, sobre un viejo estacionamiento de trenes. Está ubicado al lado de Midtown, donde los precios del suelo superan todos en el área metropolitana. Según Michael Bloomberg esto será el último y más grande proyecto urbano en Manhattan.

En principio, los monumentales edificios de Hudson Yards deberían ser más eficientes en el uso de energía, pero quedan muchas interrogantes ya que (1) las oficinas y residencias serán de muy alto costo, y muchas unidades servirán para inversión de capital y especulación. Este tipo de construcción tiene las más altas tasas de desuso por muchos períodos del año, bajando la supuesta eficiencia energética y (2) el valor del acceso al transporte público es limitado, porque el sistema subterráneo, construido hace más de un siglo ahora funciona a máxima capacidad, y hay muy pocas adiciones de estaciones y líneas. La ciudad de Nueva York ha pagado casi 3 mil millones de dólares para extender una línea menos de 
dos kilómetros hasta la nueva estación de Hudson Yards.

Hudson Yards debería ser más equitativa. Debe disminuir el déficit de unidades de vivienda en la ciudad, primero porque una cuarta parte de las unidades serán para personas de ingresos limitados y segundo porque reducirá la demanda (y las rentas) en el resto de la ciudad. El problema es que el costo de las viviendas sociales es más para la clase mediaalta que para los más necesitados y la supuesta ley de oferta y demanda nunca funciona para solucionar los problemas de escasez que afectan los que más necesitan la vivienda. Al contrario el mercado más importante en este caso es el mercado del suelo, no el mercado de la vivienda y este mercado funciona de tal manera que las nuevas construcciones en el centro de la ciudad causan graves aumentos en los precios y rentas y el desplazamiento expulsivo de los residentes más pobres.

Tal vez más problemático es el hecho que Hudson Yards formará parte de un distrito fiscal separado. Este Tax Improvement District (TIF) tendrá el derecho a fijar impuestos a medida que se vaya construyendo el proyecto y puede invertir los fondos recaudados en servicios locales como por ejemplo escuelas y parques. Es un modelo que rompe el esquema tradicional de imponer impuestos al nivel de la ciudad para entonces invertir los fondos según las prioridades del concejo comunal y el alcalde, basado en su interpretación del interés público. Es un perfecto modelo neoliberal para crear enclaves de lujo, asegurando el control de los recursos y su monopolio por los mismos privilegiados.

Espacio Público: En el espacio al sur de Hudson Yards nació hace poco The High Line, un parque hecho sobre una línea ferroviaria abandonada. Ahora es una atracción turística que ha servido para estimular la valorización de las propiedades industriales y su conversión a residencias y comercios. Ha estimulado la gentrificación del barrio de Chelsea, uno de los últimos barrios populares de Manhattan.

\section{THE WORLD TRADE CENTER, MANHATTAN}

Después de la tragedia de 2001 se inició un proceso de discusión y debate sobre qué hacer en el espacio del Bajo Manhattan donde existía el World Trade Center. Las autoridades locales y nacionales no aceptaron las propuestas para un desarrollo mínimo que favorecía el espacio público. El gobierno comprometió más de 20 mil millones de dólares para la reconstrucción de las torres y generosamente subsidió bonos ( $\mathrm{Li}$ berty Bonds) para otras construcciones verticales en Manhattan. Ahora 15 años después, una gran parte está construida. El subsidio público en la infraestructura y el desarrollo urbano será mucho mayor que el programado al inicio. Como Hudson Yards, es un enclave fuertemente subsidiado. Ahora junto con el otro gran enclave de bajo Manhattan, Battery Park City, la zona es una de las más ricas en toda la ciudad. Dado que la población residencial está creciendo en este enclave, tradicionalmente dominado por Wall Street, los urbanistas de la ciudad lo venden como una transformación desde usos divididos y separados por el sistema de la zonificación y la planificación tradicional a usos mixtos que rompe con la clásica concentración de oficinas en el centro ${ }^{18}$. En realidad no hay tanta demanda para oficinas debido a la decentralización del sector financiero que empezó décadas atrás. El histórico Chinatown, ubicado al lado del World Trade Center, perdió mucho pero 
recibió muy poca ayuda, estimulando la conversión de este enclave a un enclave más pequeño que funciona más como atractivo turístico que como barrio popular.

Espacio Público: En el caso del Zuccotti Park es significativo e irónico que uno de los movimientos más importantes del siglo presente, Occupy Wall Street, nació con la ocupación de un espacio público en propiedad privada que fue establecido en el corazón de la zona de Wall Street. Esta zona ha sido una de las más protegidas y veladas por el régimen de seguridad que fue iniciado después del 9/11. La expulsión de los ocupantes de Zuccotti Park fue coordinada por las autoridades nacionales y locales.

\section{WILLIAMSBURG, BROOKLYN}

Este es el caso más dramático de desplazamiento de un barrio en la última década. Por un siglo Williamsburg fue el modelo de un barrio residencial e industrial económicamente integrado, con usos mixtos y una población multi-étnica. Ahora es un nuevo enclave de torres de lujo. Es notable que la conversión a un barrio residencial con una población homogénea se efectuara utilizando un discurso de la integración y un plan de zonificación que pretendía crear usos mixtos pero que en realidad dio preferencia a torres residenciales ${ }^{19}$.

Durante un período de 15 años los residentes de Williamsburg presentaron un plan para el desarrollo futuro del barrio. La idea central del plan era conservar la mezcla de usos, clases sociales y etnias, evitando la concentración de torres de lujo en la costa del East River, donde la industria antiguamente dominaba. El plan fue presentado y aprobado por la City Planning Commission en 2003. Dos años después de aprobar el plan del barrio, el alcalde presen- tó y aprobó un reglamento de zonificación que tuvo el efecto de incentivar la construcción de enormes torres de lujo en la costa del East River. Después de una extendida lucha, ganó el alcalde y en un período de cinco años se llenó la costa de torres, desplazaron a más de 10000 residentes, en particular los portoriqueños y los latinos más pobres. Fue un caso de especulación y gentrificación veloz y causó un dramático aumento de alquileres con amplios abusos de las tradicionales protecciones a los inquilinos. Y 10 años después de la rezonificación, los aumentos de precios y rentas han derramado las fronteras de Williamsburg y están llegando a otros barrios de Brooklyn. La densificación se extiende y también el desplazamiento de los que tienen menos.

Williamsburg no es simplemente un caso de desarrollo independiente fuera de Manhattan sino un ejemplo de la extensión del mercado del suelo de Manhattan hasta las jurisdicciones a su alrededor. Otros ejemplos son: Long Island City (Queens), Jersey City (New Jersey) y DUMBO (Brooklyn). La escasez de terrenos disponibles en Manhattan y los precios astronómicos del suelo han exigido la búsqueda de nuevos lugares para la inversión de capital. Esto no ha sido una decentralización de la metrópolis sino más bien la extensión del centro.

Espacio Público: En 1993 se creó un nuevo régimen de zonificación para los terrenos al borde de los grandes ríos y el mar. Siguiendo el modelo de Manhattan y el modelo neoliberal de espacio público en suelo privado, esta nueva ley permitió el desarrollo de edificios de lujo en las franjas de la costa pero también garantizó que una franja entre el agua y los nuevos edificios sea dedicada a espacio público. En su implementación este modelo, usado también en Battery 
Park City, produce espacios que en la práctica sirven principalmente a los residentes de las torres de lujo.

\section{DENSIFICACIÓN DE LA VIVIENDA PÚBLICA}

En 2012, en su último año como alcalde, Michael Bloomberg presentó un plan para construir nuevas viviendas en ocho complejos de vivienda pública en Manhattan. La idea fue construir residencias privadas sobre el suelo público en medio de las viviendas sociales (public housing) ubicadas en los barrios de Manhattan -Harlem y Lower East Sid- más cercanos a Midtown y Lower Manhattan. El alcalde propuso vender los espacios públicos a inversionistas privados y con los beneficios reparar las viviendas públicas que desde la crisis fiscal de los años 70 sufrían por la baja del financiamiento público. 20\% de los nuevos apartamentos según este plan, serían dedicados a personas de bajos y medios ingresos.

El alcalde quiso vender la propuesta a los urbanistas con varios argumentos: primero, era más sustentable porque es más eficiente en el uso del suelo, la infraestructura y el transporte; segundo, ayudaba a deshacerse del diseño de la tipología de torre en el parque que interrumpía y chocaba con la famosa retícula de Manhattan que dicen, ha creado la vida dinámica de las veredas y las calles, los principales lugares de vida pública y el desarrollo de nuevos servicios comerciales, que no existen en los complejos de la vivienda pública y podría integrar los proyectos con diferentes grupos económicos (y raciales) poniendo fin a la historia de segregación racial y económica ${ }^{20}$.

Los inquilinos de los proyectos se unieron en oposición y lograron frenar la propuesta del alcalde. Me gustaría responder a los argumentos que se utilizaron a favor del proyecto. Primero, la supuesta eficiencia del uso del suelo responde a un solo criterio: el precio en el mercado del suelo. No se hizo un estudio sobre los servicios de transporte y otra infraestructura y tres de los proyectos están en una zona precaria sujeta a inundaciones. Segundo, es totalmente absurdo solucionar el problema de las torres en el parque quitando los parques y construyendo más torres, incluyendo torres dos veces más altas. Además los proyectos de Manhattan están en barrios muy compactos donde hay ya una fuerte integración espacial entre viviendas y negocios privados. Finalmente los inquilinos en oposición a la propuesta entendieron que el resultado iba a ser la construcción de apartamentos de lujo, mayormente para gente blanca e iba a crear más presiones para expulsarles a ellos, mayormente negros y latinos. Se ocultó el hecho que la vivienda pública está ya integrada, no perfectamente pero más integrada que el resto de Manhattan y ciertamente más integrada que los nuevos enclaves que son resultados del proceso de gentrificación.

Espacio Público: La propuesta fue construir nuevas torres en espacios abiertos usados para recreación y algunos espacios usados para estacionamientos.

\section{CONCLUSIONES: CONTINUACIÓN Y CRECIMIENTO DE LA METRÓPOLIS HORIZONTAL}

A pesar de la caída del mercado de vivienda en muchas áreas periféricas, y a pesar de la continuación ahora más lenta del crecimiento de los centros urbanos más densos, la metrópolis horizontal sigue siendo el hábitat para la gran ma- 
yoría de la población norteamericana. La nueva densidad central en Manhattan y los barrios a su alrededor sigue como la excepción a la regla. El área metropolitana es de baja densidad mientras el centro sigue densificando y desarrollándose a su alrededor. Manhattan y su entorno se está pareciendo más a Dubai, Las Vegas u otros enclaves exclusivos en el mundo.

Hay que tomar en cuenta no solamente el desarrollo de los megaproyectos y el crecimiento de la ciudad desigual, reconociendo que el desalojo de los residentes y de los barrios consolidados crea más presiones en el resto del área metropolitana. En muchos casos el número de los residentes expulsados del centro es más grande que los nuevos residentes, pero no sabemos quiénes son ni a donde van porque no son datos importantes en la política local. Su destino, sin duda, es la periferia metropolitana. Aunque este fenómeno está densificando ligeramente a la periferia metropolitana, ésta sigue siendo de baja densidad y viable sólo con el uso del automóvil.

No quiero cerrar este ensayo solamente con estas notas negativas. Nueva York tiene una larga historia de resistencias y luchas populares contra las expulsiones y por el derecho a la ciudad. La ciudad tiene más viviendas públicas y programas para conservar la vivienda para los que más lo necesitan que otras ciudades en USA. Pero esto es otro ensayo que tendré que dejar para el futuro.

\section{NOTAS}

1 Kunstler, J., The Geography of Nowhere, Touchstone, Nueva York, 1993.

2 El plan no es sostenible y es más un programa de corto plazo que un plan a largo plazo. Fue preparado en un período de seis meses por McKinsey Corporation, una consultora externa, utilizando un estudio sobre terrenos baldíos e industriales de supuesto interés para inversionistas y desarrolladores. El plan nunca fué aprobado pero ha servido como guía para la política del suelo urbano. Veáse Angotti, T., Is New York's Sustainability Plan Sustainable?, ACSP/AESOP, Chicago, 2008.

3 Glaeser, Edward 2011 Triumph of the City, Penguin, Nueva York, 2011 y Owen, David Green Metropolis, Riverhead, Nueva York, 2009.

4 Gallagher, Leigh, The End of the Suburbs, Penguin, Nueva York, 2004.

5 City of New York, Hazard Mitigation Plan http://www.nyc. gov / html / oem / htm l/ planning response / planning hazard mitigation.shtml, 2014.

6 Jacob, Klaus, Manhattan Will need 'Venice-like Canals to Stop Flooding, Next City, http://nextcity.org/daily/entry/ climate-scientist-manhattan-needs-venice-like-canalsflooding, 2014. Accedido en 7/4/14.
7 City of New York, Inventory of New York City Greenhouse Gas Emissions, PlaNYC. Environmental Defense 2007, All Choked Up, www.allchokedup.org, 2011.

8 Garreau, Joel, Edge City, Doubleday, Nueva York, 1991.

9 Environmental Defense 2007 All Choked Up. www.allchokedup.org

10 Pratt Center for Community Development, Transportation Equity Atlas. NY. http://prattcenter.net/research/ transportation-equity-atlas, 2010.

11 Harvey, David, Rebel Cities,Verso, Londres, 2012, 42-5.

12 Angotti, Tom, New York for Sale, MIT Press, Cambridge, 2008.

13 Mathias, Christopher, These maps show just how segregated New York City really is, Huffington Post, April 14, 2014. http://www.huffingtonpost.com/2014/04/15/newyork-city-segregation-map_n_5153739.html. Roberts, Sam, Manhattan's Income Gap is Widest in US Census Finds, New York Times, September 18, 2014.

14 Brash, Julian, Bloomberg's New York, University of Georgia, Athens, 2011.

15 Angotti, Tom, Rethinking the Luxury City, The Indypendent. December, 2013. https://indypendent.org/2013/12/17/ 
rethinking-luxury-city

16 Zukin, Sharon, Naked City, Oxford, Nueva York, 2010.

17 Picture the Homeless, Banking on Vacancy, Nueva York, 2012.

18 Veáse op. cit. nota 12, 182-86.
19 Angotti, Tom, Zoning Without Plannin, http://tomangotti.files.wordpress.com /2014/04 /zoning-without-planning-2014.pdf, 2014.

20 Center for Community Planning \& Development, Keeping the Public in Public Housing, Hunter College, http:// www.hunter.cuny.edu/ccpd, Nueva York, 2014.

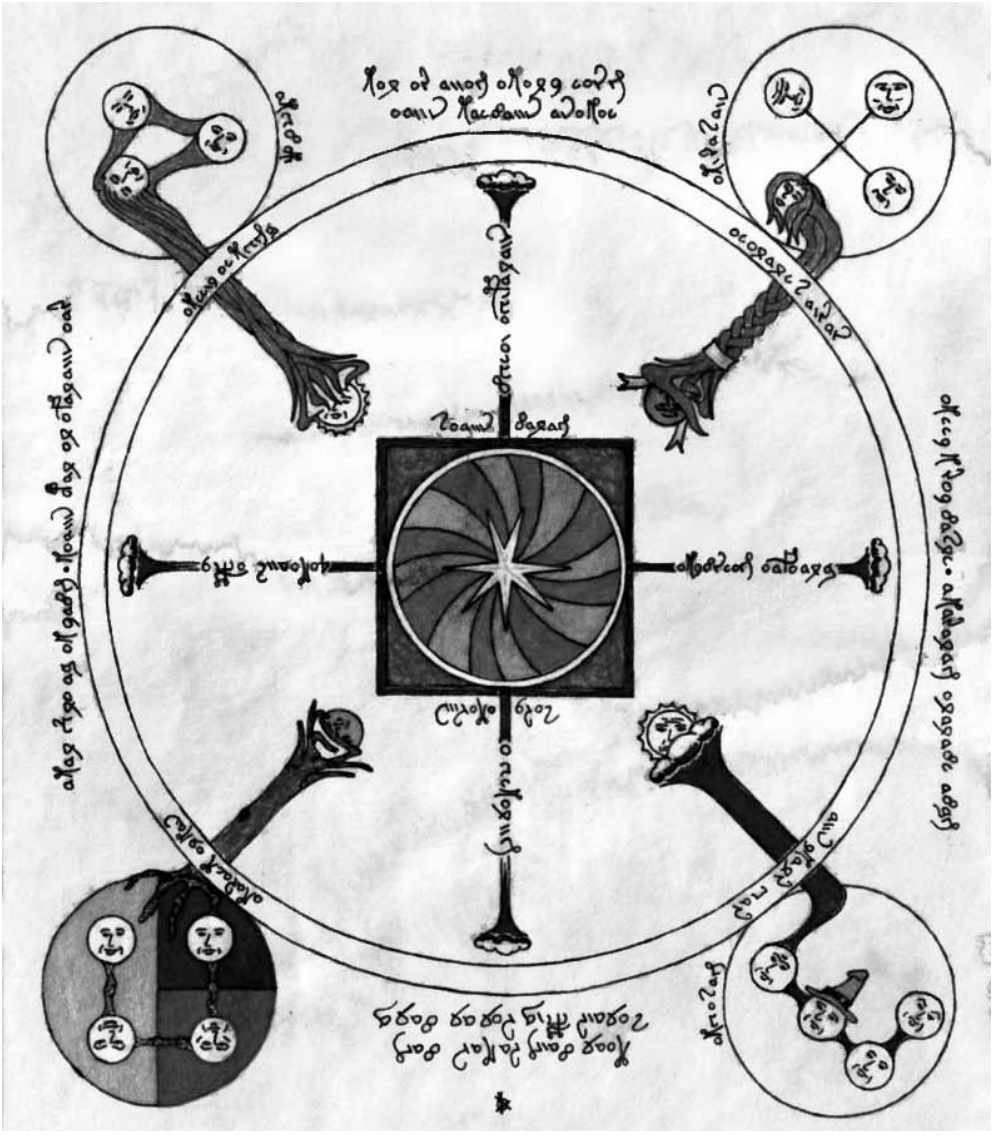

